\title{
A New Ultrasound Biomicroscopic Sign seen after Deep Sclerectomy (Dolphin Head Sign)
}

\author{
${ }^{1}$ Ahmed M Abdelrahman, ${ }^{2}$ Hala M El Cheweikh, ${ }^{3}$ Dina MS El-Fayoumi, ${ }^{4}$ Riham SHM Allam
}

\begin{abstract}
Purpose: To describe a new ultrasound biomicroscopic (UBM) sign seen in patients who underwent deep sclerectomy (DS) as a surgical procedure for the management of uncontrolled primary open-angle glaucoma (POAG). The presence of this sign in ultrasound biomicroscopy is suggested to be an indicator of successful surgery. We would like to name this sign as the "dolphin head sign."
\end{abstract}

Design: Prospective interventional study.

Materials and methods: Twenty-eight eyes of 17 patients with POAG underwent DS with intraoperative mitomycin C (MMC) $0.3 \%$ applied for 2 minutes under the superficial scleral flap. Patients were followed up for a minimum of 6 months. Ultrasound biomicroscopy was done at the third postoperative month to evaluate the surgical area in both successful and failed cases.

Results: The study included 28 eyes of 17 patients. The mean age of the study group was $42.90 \pm 14.37$ years (20-64 years). The study included 10 females and 7 males. The mean preoperative intraocular pressure (IOP) was $24.57 \pm 6.37 \mathrm{~mm} \mathrm{Hg}$ (20-38 mm Hg). The mean best corrected visual acuity (BCVA) was $0.57 \pm 0.3(0.05-1.00)$. Complete success has been achieved in 21 eyes (75\%) during the follow-up period, with a mean IOP of $12.00 \pm 3.86 \mathrm{~mm} \mathrm{Hg}(6-20 \mathrm{~mm} \mathrm{Hg})$. The dolphin head sign was demonstrated only in successful cases, whereas the unsuccessful cases failed to show the typical sign.

Conclusion: The presence of a "dolphin head" configuration in UBM images could be taken as an indicator of successful DS.

Keywords: Deep sclerectomy, Dolphin head sign, Glaucoma, Glaucoma surgery, Ultrasound biomicroscopy.

How to cite this article: Abdelrahman AM, El Cheweikh HM, El-Fayoumi DMS, Allam RSHM. A New Ultrasound Biomicroscopic Sign seen after Deep Sclerectomy (Dolphin Head Sign). J Curr Glaucoma Pract 2016;10(2):56-59.

Source of support: Nil

Conflict of interest: None

\section{INTRODUCTION}

In the 1990s, nonpenetrating glaucoma surgery (NPGS) like viscocanalostomy and deep sclerectomy (DS) were introduced as safer alternatives to trabeculectomy. ${ }^{1,2}$

\footnotetext{
${ }^{1,2}$ Professor, ${ }^{3,4}$ Lecturer

${ }^{1-4}$ Department of Ophthalmology, Kasr Al-Ainy School of Medicine, Cairo University, Giza, Egypt

Corresponding Author: Dina MS El-Fayoumi, Lecturer Department of Ophthalmology, Kasr Al-Ainy School of Medicine Cairo University, Giza, Egypt, Phone: +21006044030, e-mail: d_elfayoumi@yahoo.com
}

The main difference between NPGS and trabeculectomy is the creation of a filtration membrane: The trabeculo-Descemet's membrane (TDM) instead of a sclerostomy. Excision of the inner scleral flap during NPGS will create a subscleral lake (intrascleral bleb) where the aqueous collects. The postsurgical outflow pathways of aqueous remain unclear, but increased flow through the Schlemm's canal, suprachoroidal space, and subconjunctival flow with "bleb" formation have all been postulated. ${ }^{3-6}$ Most reports agree that NPGS has a lower rate of complications than trabeculectomy. ${ }^{7-9}$ Deep sclerectomy has been shown to be of equal results to trabeculectomy in lowering the intraocular pressure (IOP). ${ }^{10,11}$ Similarly to trabeculectomy, intraoperative mitomycin C (MMC) application results in lower IOP. ${ }^{12}$

Ultrasound biomicroscopic (UBM) studies in human eyes that underwent DS have shown formation of a subscleral lake, an overlying bleb, and a supraciliary hypoechoic area. ${ }^{13}$

In our study, we noticed that the UBM appearance of the surgical area simulates the "dolphin head" in successful cases. Accordingly, we named the configuration of the surgical area as the "dolphin head sign."

The aim of the present work is to describe this new sign seen with UBM during evaluation of eyes with DS and to correlate its presence with surgical success of the cases.

\section{MATERIALS AND METHODS}

\section{Study Subjects}

This is a prospective interventional study including 28 eyes of 17 patients having primary open-angle glaucoma (POAG) for which DS was done. The study subjects were recruited from Kasr Ainy subspecialty glaucoma clinic in the period starting from November 2013 to March 2014. The patients were followed up for a minimum period of 6 months. They were enrolled in this study after giving a written informed consent to participate and the Tenets of the Declaration of Helsinki were followed.

Deep sclerectomy was performed under the standard peribulbar anesthesia. Fornix-based conjunctival incision was done followed by a $4 \times 3 \mathrm{~mm}$ rectangular superficial 
scleral flap using a crescent blade. Dissection was done until we got adequate exposure of the corneal periphery. Mitomycin C soaked sponges ( $0.3 \%$ for 2 minutes) were applied under the superficial scleral flap and the conjunctiva. Deep flap dissection was then carried out with deroofing of Schlemm's canal. Percolation was then tested using sponges and the deep flap was excised. The superficial flap was then sutured using 10/0 nylon sutures one at each corner of the flap. The whole operations were performed by a single experienced surgeon (AA).

The patients were followed up for the IOP, best corrected visual acuity (BCVA) as well as full ophthalmological assessment. Ultrasound biomicroscopy was done in the third postoperative month to evaluate the surgical area and compare its appearance to IOP control.

Ultrasound biomicroscopy was done by a single experienced investigator (HE) using a UBM $50 \mathrm{MHz}$, Ophthalmic Technologies Inc; Canada (OTI) ultrasonic scan images. With the patient in the supine position, and with the aid of an emersion scleral shell and an examination gel, the surgical area was scanned with the UBM probe. Radial and transverse sections of the sclerectomy area at 12 o'clock were explored using optimal $\mathrm{dB}$ gain that allowed best resolution and quality of images.

Statistical analysis was performed using Statistical Package for the Social Sciences (SPSS) for Windows (SPSS Inc, Chicago, Illinois, USA). The statistical data were expressed in terms of mean, range, and statistical deviation.

\section{RESULTS}

Our study was performed on 28 eyes of 17 patients who underwent DS with MMC between November 2013 and March 2014 at the Department of Ophthalmology, Kasr Al-Ainy School of Medicine, Giza, Egypt.

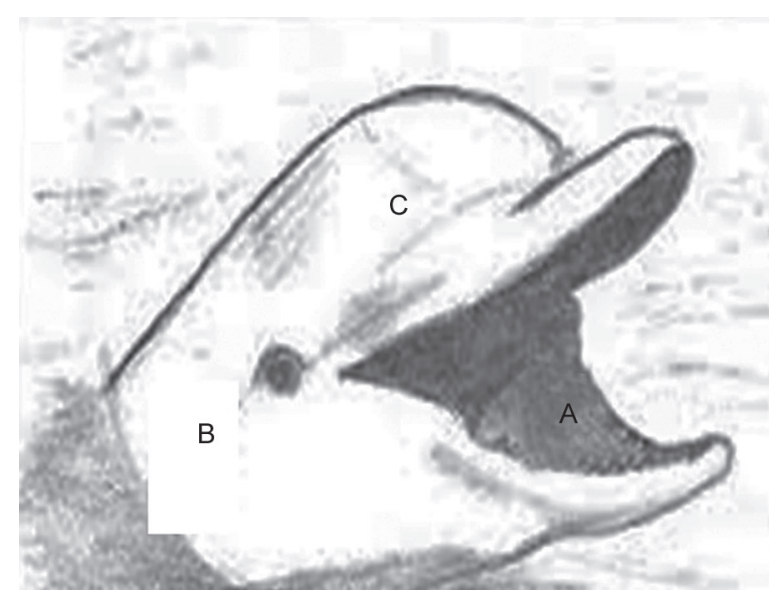

Figs $1 \mathrm{~A}$ to $\mathrm{C}$ : The head of the dolphin (left) compared to the ultrasound biomicroscopic appearance in deep sclerectomy (right): (A) Mouth of the dolphin compared to anterior chamber angle in UBM; (B) eye of the dolphin compared to intrascleral lake; and $(C)$ head of the dolphin compared to filtering bleb
Table 1: Preoperative and demographic data of successful cases

\begin{tabular}{lll}
\hline & Range & Mean $\pm S D$ \\
\hline Age & $20-64$ years & $42.90 \pm 14.37$ \\
Gender & $7 \mathrm{~F}: 5 \mathrm{M}$ & $58.3 \%: 41.6 \%$ \\
Preoperative medications & $1-4$ & $2.9 \pm 0.2$ \\
Preoperative IOP & $20-38 \mathrm{~mm} \mathrm{Hg}$ & $24.57 \pm 6.37 \mathrm{~mm} \mathrm{Hg}$ \\
BCVA & $0.05-1.00$ & $0.57 \pm 0.3$ \\
OD/OS & 11 OD:10 OS & $52.4 \%: 47.6 \%$ \\
\hline
\end{tabular}

All patients underwent DS with the adjuvant use of MMC $(0.3 \%$ applied for 2 minutes), and the patients were followed up for a period of 6 months during which a UBM was performed in the third postoperative month to assess the surgical area.

During the study period, 21 eyes (75\%) achieved IOP control without the need for adjuvant therapy (complete success; IOP $<21 \mathrm{mmHg}$ without medications or interventions) with a mean IOP value of $12.00 \pm 3.86 \mathrm{~mm} \mathrm{Hg}$ (6-20 mm Hg) (Table 1).

Seven eyes $(25 \%)$ of five patients did not show criteria of surgical success with persistently high IOP above $22 \mathrm{~mm} \mathrm{Hg}$ (mean IOP value of $24.8 \pm 0.73 \mathrm{~mm} \mathrm{Hg}$ ) that required control using two topical antiglaucoma medication in four eyes and subscleral trabeculectomy in one eye.

Ultrasound biomicroscopy was done for both successful and failed cases. The dolphin head has been demonstrated in all the successful cases (Figs 1 and 2). The mouth of the dolphin was formed by the anterior chamber angle, the eye of the dolphin corresponded to the Intrascleral lake, and the vault of the head corresponded to the filtering bleb.

In failed cases, we noticed that collapse of the intrascleral lake and or disappearance of the filtering bleb will lead to either a disfigured dolphin head or even its total disappearance (Figs 3A and B).

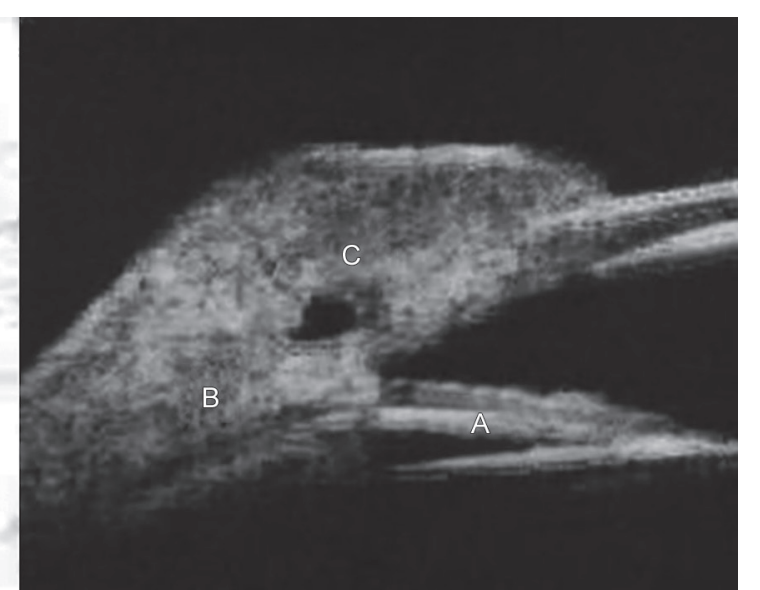



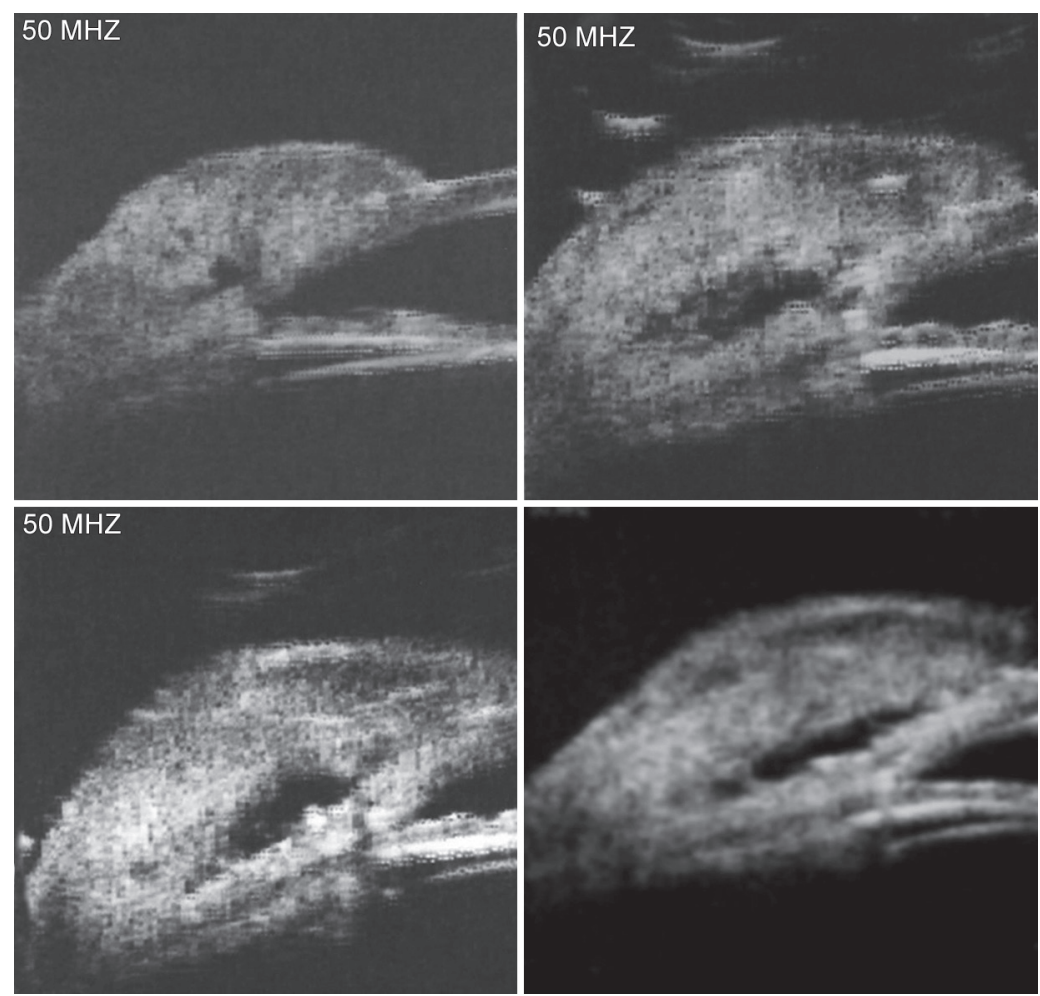

Fig. 2: Ultrasound biomicroscopic radial scan revealing a sonoluscent area between deep scleral lamellae "intrascleral lake" (decompression chamber) showing the dolphin head appearance
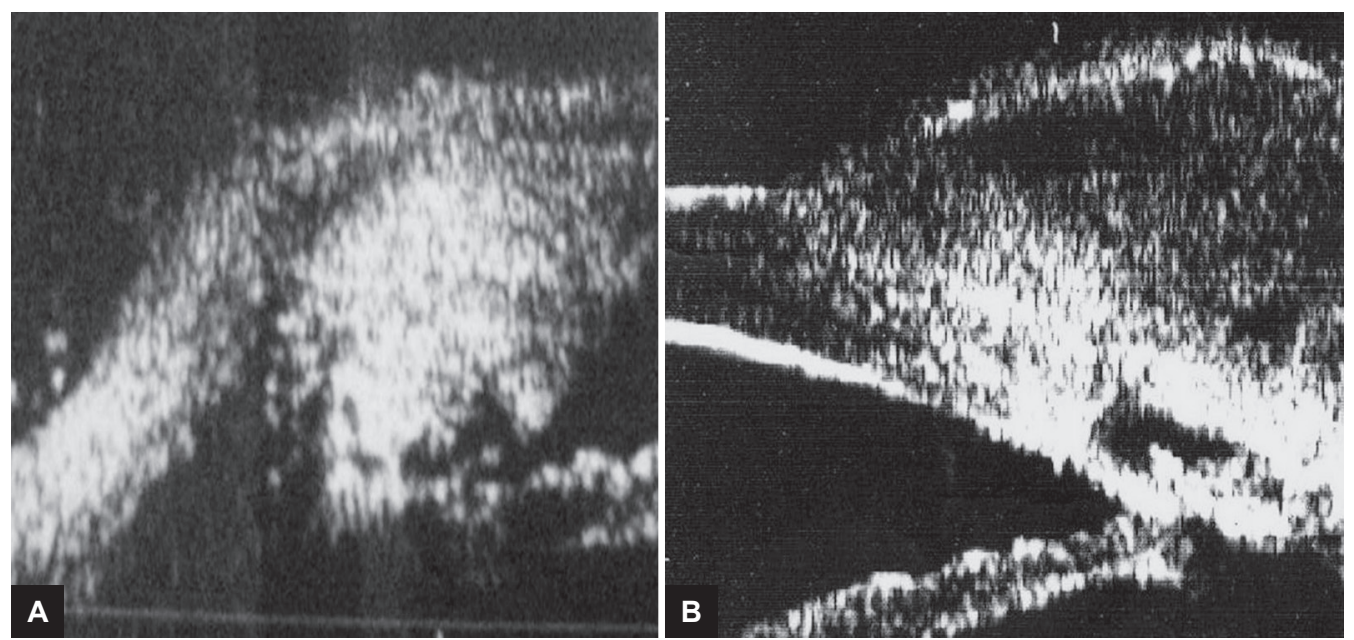

Figs 3A and B: (A) Ultrasound biomicroscopic radial scan revealing the absence of the typical "dolphin head" in failed cases vs (B) its presence in successful cases

\section{DISCUSSION}

Ultrasonic biomicroscopy allows structural examination after performing filtering surgery with interpretation similar to that shown in histological sections. ${ }^{14}$ It also provides details of the anterior chamber, ciliary body, and corneoscleral limbus - areas that cannot be examined using other methods in the living eyes. ${ }^{15}$

Ultrasound biomicroscopy is useful in the assessment of anterior chamber angle structures and for evaluating the surgical area after performing various interventions to treat glaucoma. A valuable use of UBM is to assess the filtration area after glaucoma surgeries, penetrating or nonpenetrating, and trying to correlate IOP control to bleb criteria.

Yamamoto et $\mathrm{al}^{16}$ used UBM images to further elucidate the internal structure of filtering bleb and were able to establish a new system for classifying filtering blebs based on bleb reflectivity. They also correlated this with IOP control. Blebs were classified into four groups - type L (low reflective) blebs showed good IOP control, with 
moderate-high bleb height and identifiable microcysts; type $\mathrm{H}$ (high reflective), type $\mathrm{F}$ (flattened), and type $\mathrm{E}$ (encapsulated) were associated with poor IOP control. Other UBM findings reported in literature include progressive reduction in the Intrascleral lake and its eventual collapse. ${ }^{17}$

In our study, we describe a new UBM appearance that could be a clue to successful DS. That appearance of the surgical area can be simulated to a dolphin's head. The observation of this sign should be taken into consideration during patients' evaluation and can be considered as a criterion of success as this sign was not elicited in failed cases.

Our study is a short-term one that describes a characteristic appearance of the surgical area during the early postoperative period (3-6 months). Longer follow-ups with serial imaging are required to describe a change in this morphology with time. The present study is limited by the relatively small sample size which can be attributed to the fewer cases which undergo DS and also a relatively short follow-up period.

\section{CONCLUSION}

Presence of a dolphin head configuration in UBM images is suggested to be an indicator of successful surgery in patients undergoing DS.

Financial Disclosure: The authors have no financial interests in the material discussed in this work.

\section{REFERENCES}

1. Stegmann R, Pienaar A, Miller D. Viscocanalostomy for openangle glaucoma in black African patients. J Cataract Refract Surg 1999 Mar;25(3):316-322.

2. SanchezE,SchnyderCC,Sickenberg M,Chiou AG,HédiguerSE, Mermoud A. Deep sclerectomy: results with and without collagen implant. Int Ophthalmol 1996;20(1-3):157-162.

3. Lei J, Sun N, Zhao X, Kang Q, Chen L, Fan X. Morphologic study of the drainage pathway using a tracer after a bypass filtering procedure in rabbit eyes. Ophthalmic Surg Lasers Imaging 2011 May-Jun;42(3):254-262.

4. Xu W, Yao K, Wu W, Li Z, Ye P. Change in outflow pathway of porcine eyes in vitro by non-penetrating filtering surgery. Can J Ophthalmol 2010 Dec;45(6):632-636.

5. Aptel F, Dumas S, Denis P. Ultrasound biomicroscopy and optical coherence tomography imaging of filtering blebs after deep sclerectomy with new collagen implant. Eur J Ophthalmol 2009 Mar-Apr;19(2):223-230.

6. Delarive T, Rossier A, Rossier S, Ravinet E, Shaarawy T, Mermoud A. Aqueous dynamic and histological findings after deep sclerectomy with collagen implant in an animal model. Br J Ophthalmol 2003 Nov;87(11):1340-1344.

7. Carassa RG, Bettin P, Fiori M, Brancato R. Viscocanalostomy versus trabeculectomy in white adults affected by openangle glaucoma: A 2-year randomized, controlled trial. Ophthalmology 2003 May;110(5):882-887.

8. Cillino S, Di PF, Casuccio A, Cillino G, Lodato G. Deep sclerectomy versus trabeculectomy with low-dosage mitomycin C: Four-year follow-up. Ophthalmologica 2008;222(2): 81-87.

9. O'Brart DP, Rowlands E, Islam N, Noury AM. A randomised, prospective study comparing trabeculectomy augmented with antimetabolites with a viscocanalostomy technique for the management of open angle glaucoma uncontrolled by medical therapy. Br J Ophthalmol 2002 Jul;86(7):748-754.

10. Yarangumeli A, Gureser S, Koz OG, Elhan AH, Kural G. Viscocanalostomy versus trabeculectomy in patients with bilateral high-tension glaucoma. Int Ophthalmol 2004 Jul;25(4):207-213.

11. Shaarawy T, Mermoud A. Deep sclerectomy in one eye vs deep sclerectomy with collagen implant in the contralateral eye of the same patient: Long-term follow-up. Eye (Lond) 2005 Mar;19(3):298-302.

12. Kozobolis VP,ChristodoulakisEV,TzanakisN,ZacharopoulosI, Pallikaris IG. Primary deep sclerectomy versus primary deep sclerectomy with the use of mitomycin $C$ in primary open-angle glaucoma. J Glaucoma 2002 Aug;11(4):287-293.

13. Kazakova D, Roters S, Shnyder CC, Achache F, JonescuCuypers C, Mermoud A, Krieglstein G. Ultrasound biomicroscopy images: Long term results after deep sclerectomy with collagen implant. Graefes Arch Exp Ophthalmol 2002 Nov;240(11):918-923.

14. Sánchez Salorio M, García Feijóo J, Pazos González B. Biomicroscopía ultrasónica en oftalmología. Madrid: Tecnimedia Editorial; 1998.

15. Pavlin C, Harasiewicz K, Sherar M, Foster FS. Clinical use of ultrasound biomicroscopy. Ophthalmology 1991 Mar;98(3):287-295.

16. Yamamoto T, Sakuma T, Kitazawa Y. An ultrasound biomicroscopic study of filtering blebs after Mitomycin C trabeculectomy. Ophthalmology 1995 Dec;102(12):1770-1776.

17. Negri-Aranguren I, Croxatto O, Grigeria DE. Midterm ultrasound biomicroscopy finding in eyes with successful viscocanalostomy. J Cataract Refract Surg 2002 May;28(5):752-757. 